epigastrium). His medical attendant agreed with me in thinking that if any further bleeding occurred an operation was advisable. He continued to improve for a week, then he had another attack of collapse, evidently another intestinal haemorrhage. The surgeon who was called in found him too ill to bear an operation; he died soon afterwards from this, the third, bleeding. I have no doubt that in this case the haemorrhage was due to an ulcer of the duodenum.

Case III.-A short time ago I saw in consultation a gentleman, aged 35, who for fifteen years had suffered from recurring attacks of dyspepsia; pain in the stomach, coming on again at a considerable interval after taking food, and lasting some hours, relieved by the introduction of food into the stomach; no vomiting; no flatulence. On several occasions these attacks were attended with tarry motions, and on one occasion with vomiting of blood. These attacks only occurred in the winter, and were apparently due to exposure to cold ; the patient's occupation exposed him to cold and wet. He always was better, and free from the attacks during summer and warm weather. I saw him just as he was recovering from a severe attack. There was nothing to be made out on examination. I agreed with his medical attendant in thinking that the case was one of ulcer of the duodenum. We advised that as soon as he was able to travel he should go to Egypt, in order to get into the sun; as I have already said, he always got better in warm weather. Unfortunately that prescription could not be carried out; he went on well for a week, and then suddenly died from very acute haemorrhage.

Now these three cases of duodenal ulcer, in all of which the patients died from haemorrhage, have made a very profound impression on my mind; they show that in cases of duodenal ulcer the risk of death from haemorrhage is very great; they seem to me to suggest that one should advise an operation as soon as one makes a definite diagnosis of duodenal ulcer, provided, of course, that the patient is in a condition to be operated upon. I am convinced that the risk of fatal haemorrhage is very much greater in cases of duodenal ulcer than in cases of gastric ulcer, at all events, gastric ulcer in young chlorotic women. In cases of duodenal ulcer there is also the risk of perforation and fatal peritonitis. I have seen several cases of that sort.

Case IV.-The fourth patient, a gentleman, aged 37, I saw in consultation about a month ago. For four years he had suffered from the same group of symptoms-recurring attacks of severe pain in the epigastric region, the pain occurring a considerable time after eating, and relieved by taking food; no vomiting; no flatulence. On several occasions he had had melaena, and on one or two occasions very profuse vomiting of blood. I diagnosed a duodenal ulcer, and advised that he should be operated on without delay. There was no ulcer in the anterior wall of the duodenum; one cannot get to the posterior surface. A communication was established between the stomach and intestine (gastro-enterostomy). The patient has done well.

In conclusion, let me say that in cases of acute peritonitis due to the perforation of a gastric or duodenal ulcer immediate operation is imperative; every hour that passes, that is put off, diminishes the patient's chances of recovery. I am strongly convinced from personal experience that in cases of this kind the patient's chance of recovery largely depends upon two things in particular -one is early operation and the other is quick operation; every hour that elapses between the occurrence of the perforation and the operation diminishes the risk of recovery, and the quicker the operation (other things being, of course, equal) the greater the chances of recovery. If I were to develop peritonitis in consequence of the rupture of a gastric or duodenal ulcer, I would have an operation at the earliest possible moment, and I would ask the surgeon to perform the operation as quickly as possible.

UNDER the will of the late Mr. Vyell Edward Walker, formerly a famous amateur cricketer, who died on January 3rd, the London Hospital and the Cancer Hospital each receive $£ 2,000$ and the Middlesex Hospital, the City of London Hospital for Diseases of the Chest, Victoria Park, the Royal Hospital for Incurables, Putney, the Poplar Hospital for Accidents, the Idiot Asylum, Earlswood, the Hospital for Consumption, Brompton, and the Victoria Hospital for Sick Children, sums of $£ 1,000$ each.

\section{A Cliniral lerture}

\section{THE SURGICAL TREATMEN'T OF GASTROPTOSIS,}

\author{
WITH AN ACCOUNT OF FIVE CASES.*
}

\section{By FREDERIC EVE, F.R.C.S.,} SURGEON TO THE LONDON HOSPITAL.

Gastroptosis, or falling of the stomach, may be associated with Glenard's disease, or falling of all the abdominal viscera, with mobility of the right kidney, or it may exist alone. The condition depends mainly on stretching of the suspensory ligaments of the stomach -namely, the gastro-phrenic ligament and gastro-hepatic omentum. It may be further exaggerated by displacement downwards of the liver, to which the latter structure is attached.

Gastroptosis is by no means uncommon, Glenard himself having observed it 400 times among 1,300 patients. The causation is obscure, but its chief basis is probably anatomical. The subjects are usually women the lower orifice of whose thorax is narrow, the dome of the diaphragm low, and the costal margins diverging less obliquely than usual. Tight-lacing, by producing this condition, is undoubtedly an important factor. The muscular parietes of the abdomen are as a rule lax, the muscles ill-developed, and sometimes there is separation of the recti. This condition may have been increased by pregnancy. Loss of fat and consequent diminution of intra-abdominal pressure is a determining cause. In one reported case the omentum was firmly adherent in the pelvis, and in one of my own cases there were adhesions between the anterior wall of the stomach and the parietes. The right kidney is usually movable. In two cases at present under my care this peculiarity exists.

Neurasthenia, often in a high degree, is almost invariably present, and materially adds to the difficulties of treatment. It is not my intention to discuss the relation of neurasthenia to the physical condition; but I have little doubt that in many cases the neurosis is secondary and the result of malnutrition following on the disturbance of the functions of the alimentary canal.

\section{Sумттомs.}

Dr. Beyea well sums up the symptoms when he says : "The picture is that of chronic severe atonic dyspepsia with neurasthenia and increasing emaciation."

The patient complains of severe chronic dyspepsia of long standing, with eructations, sense of fullness and weight at the epigastrium and pain after food. In the more severe cases she is unable to take meat or even solid food; there is vomiting, either slight or only of mucus, in attacks at infrequent intervals, and, in the worst cases where considerable dilatation of the stomach has occurred, the vomiting is frequent and copious. Severe lumbar pains may be complained of. In two of the cases quoted below there was a tender spot on pressure over the epigastrium, and in two a history of haematemesis. In my first case the functions of the stomach were so much altered that free hydrochloric acid was absent from a test meal.

Physical examination shows displacement downwards of the stomach, the lesser curvature being situated in the neighbourhood of the umbilicus, just above it, or even well below it. The pancreas can be felt above the lesser curvature of the stomach, a condition on which Bertrand Dawson ${ }^{1}$ has laid stress. The stomach is often dilated as well as displaced, and splashing may be obtained. Lavage in one of my cases proved distinct gastrectasis to exist.

The coincidence of mobility of the kidney may raise a question as to the part respectively played by the stomach and kidney in the production of the symptoms. The seat of the pain and the character of the symptoms will usually define which organ is mainly responsible. $\mathrm{Re}$ ported cases, however, show that nephropexy has been first performed without relief to symptoms; on the other hand, Dr. F. J. Smith informs me that in two cases under his care, believed to be suffering from gastroptosis, the

* Part of a clinical lecture delirercd at the London Hospital. 
symptoms were entirely relieved by fixation of the kidney.

Allusion has already been made to the occasional coexistence of hepatoptosis, and owing to the form of the thorax a Riedel's lobe is commonly present.

\section{Cases.}

The following is a brief account of the patients upon whom I have operated. With one exception, all had been previously under the care of my colleague, Dr. Bertrand Dawson, who had exhausted the resources of medical treatment, and to whose notes I am indebted for results of the medical investigation of the cases. He was also present and conferred with me at the operations.

Case I.

Elizabeth C., aged 50, was admitted on August 3rd, 1903, having been treated in the medical wards for about six weeks. Her symptoms had existed for ten years. They consisted of pain after food, vomiting of frothy phlegm soon after food, but no return of the food itself. Four years ago she brought up about a quart of black coffee-like material. No gastrectasis existed, but there was tenderness on deep pressure in the existed, but there was tenderness on deep pressure in the
epigastrium. The lesser curvature was only just above the umbilicus, and the stomach was dilated after inflation. A test meal showed that free hydmochloric acid was absent, but that butyric and lactic acid were present.

\section{Operation.}

The following operation was performed on August 4th, 1903 The dependent greater curvature was raised and fixed by several silk sutures to the anterior abdominal parietes, about 2 in. above the umbilicus. The stitches were passed through the great omentum at its attachment to the stomach and also through the stomach wall, and then through the and also through the stomach wall, and then through the was also raised by passing some sutures between the lesser curvature and the under surface of the liver.

\section{After-history.}

Improvement followed the operation; there was considerably less pain, less flatulence and sickness. A year after the operation Dr. Cuthbert, of Croydon, wrote me: "Mrs. C.'s life has been quite bearable since the operation ; it was not before."

It is probable that in this case the secretory functions of the stomach had been greatly damaged by long continued chronic gastritis, as evidenced by the absence of free hydrochloric acid from a test meal.

CASE II.

Jane B., aged 30, had suffered from severe indigestion for fourteen years, never being able to take meat; at intervals the symptoms were more acute, pain being increased by food and often followed by vomiting. After the birth of her first child, eight years ago, her symptoms became more marked. She occasionally hrought up blood, but never more than two tablespoonfuls. Latterly she has only been able to take soda and milk, and even this was followed by pain and vomiting. milk, and even this was followed by pain and vomiting. Free hydrochloric acid was present, but no lactic acid. No
gastrectasis. The lesser curvature was $1_{\frac{1}{2}}$ in. above the umbilicus, and the greater curvature $2 \frac{1}{2}$ in. below it. The pancreas could be clearly felt above the lesser curvature.

\section{Operation.}

An operation was performed on April 29th, 1904. There were adhesions of the anterior wall of the stomach, near the lesser curvature, to the left lobe of the liver and to the abdominal wall. The lesser curvature was on a level with the umbilicus, and the liver was prolapsed.

The gastro-hepatic omentum was extremely stretched, and so thin that it would not hold a stitch. The feasibility of raising the lesser curvature of the stomach by running puckering sutures through the gastro-hepatic omentum was discussed, and discarded as impracticable. Pushing up the liver, however, was found to raise the stomach, and therefore the first step in the operation consisted in raising and fixing the liver by four strong silk sutures passed through its anterior surface and edge, and then through the anterior abdominal parietes, just below the costal margin. The greater curvature was then raised, and attached by two sutures to the anterior abdominal parietes above the umbilicus.

\section{After-history.}

I saw her fifteen months after the operation, and she reported herself greatly benefited. Before the operation she was constantly laid up with pain and vomiting; now she is able to do her housework, and can digest fish but not meat.

It may be stated that the two operations described above were performed on the spur of the moment, to meet the condition found on opening the abdomen, and I was not then aware that somewhat similar operations had been previously performed.

Kate B., aged 38, began to suffer from indigestion twenty years before admission. Two years subsequently she had an attack of haematemesis to the extent of $1 \frac{1}{2}$ pints, and a few months later of melaena. Ever since she bas suffered severely from indigestion, and has pain, especially after meals, with frequent nausea, but rarely vomiting. Hard exercise made the pain worse. The lesser curvature occupied a position just above the umbilicus, and just above the umbilicus, and the pancreas could be felt above able ; the lower aperture of the thorax was extremely narrow.

\section{Operation.}

On opening the abdomen, February 9th, 1906, the lesser curvature was found to be an inch above the umbilicus and the stomach not materially the stomach not materially sign of ulcer, and the pylorus sign of ulcer, and the pylorus
was natural to touch, although was natural to touch, although
somewhat suddenly bent on somewhat suddenly bent on
itself at its junction with the itself at its junction with the
duodenum. The lesser omentum consisted of a membrane so thin that it would scarcely bear touching The left lobe of the liver was prolonged downthe liver was prolonged downwards (see Fig. 1). A sand-bag was placed beneath the back and the liver raised and held
upwards (Fig. 2) as in performupwards (Fig. 2) as in performing operations on the gall then passed through the lesser curvature of the stomach

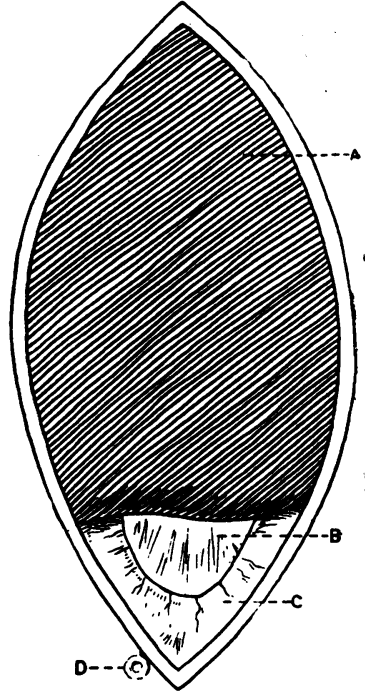

Fig. 1.-Condition on opeuing abdomen with liver in position. A, liver; B, small omentum ; c,
stomach ; D, umbilicus. ranging from the pylorus to the cardiac orifice, and above omentum to the liver, omentum to the liver, and forwards, somewhat deeply, through itself, in order to obtain a better hold (Fig. 3).

When these sutures were tied the lesser curvature was effectually raised (Fig. 4), and the greater curvature was roughly 2 in. above the umbilicus. There being a tendency to displacement of the liver downwards, a series of interrupted sutures were carried through the anterior surface the left lobe of of the liver, and then through the margins of the costal cartilages. It to expose the right lobe of the liver without making a transverse incision along the costal margin and this was not thought this was not thought lobe seemed to be partly instrumental in partly instrumental in pushing the stomach downwards. The pital a month after

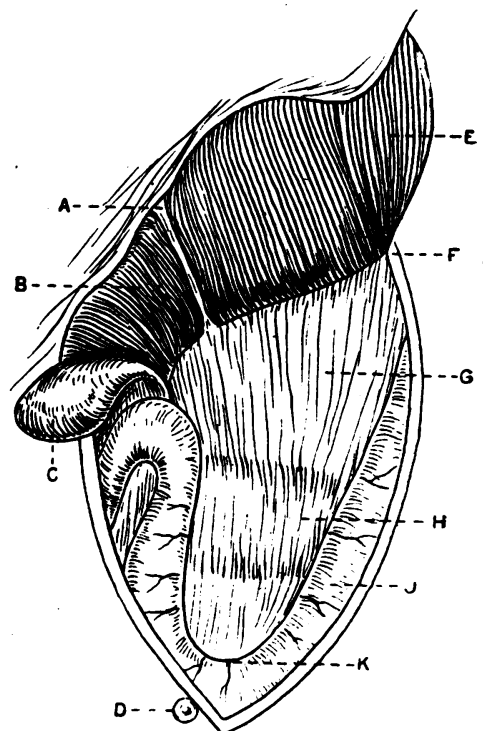

Fig. 2.-Condition when liver turned up. Liver held up and partially covered by gauze. $\mathbf{A}$, round ligament; $\mathbf{3}$, quadrate obe; c, gall bladder ; D, umbilicus , liver-left lobe; $F$, transverse fissure , pancreas seen through small omentum J. stomaclr; $\mathbf{k}$, lesser curvature 1 iu, above umbilicus. the operation, and

stated that she felt quite well; her future progress will be watched.

I regard this as by far the most satisfactory operation of its kind $I$ have performed in regard to the position of the stomach after the operation; and I thini it superior to the operation performed by Beyea, on the grounds that the gastro-hepatic omentum is so stretched in many cases as to be unsuitable for the application of sutures. On the other hand, the firm liver substance, just in front of the attachment of the lesser omentum, constitutes an admirable medium for suturing, and the lesser curvature of the stomach is scarcely altered from its natural relationships.

CASE IV.

Agnes F., agen 34, was admitted to the London Hospital February 12th, 1903. She had suffered from indigestion for three years. There was pain coming on immediately after food ; vomiting, which relieved the pain, and latterly she bad vomited increasingly larger quantities. Often articles of food, 
such as currants, which had been taken a day or two previously, were noticed in the vomit. Hacmatemesis had occurred three or four times, the largest amount being half a pint. Free hydrochloric acid was present, and lavage showed distinct gastrectasis.

The lesser curvature of the stomach was situated at the junction of the lower third with the upper two-thirds of a line between the ensiform cartilage and the umbilicus; the

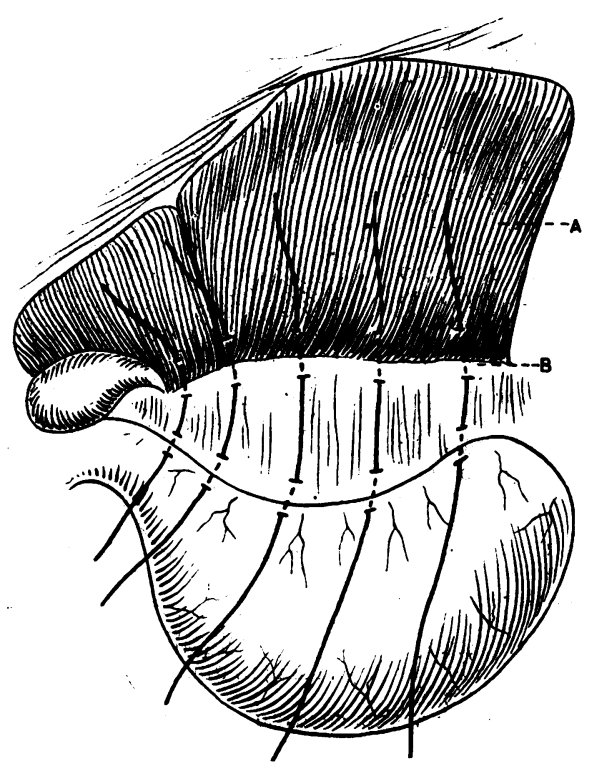

Fig. 3.-A, liver: B, junction of gastro - hepatic omentum with liver. greater curva ture between the umbilicus the rmbilicus and the pubes. readily obreadily

Operation.

At thenperation on February 13th, 1903, was displaced, consider a b l y dilated, and distinetly atonic. There was no evidence of ulcer, and the pywas patent. Posgastroentero s to $\mathrm{m}$ y was performed in the usual way.

After-history.

I sawher two years and five months after the operation.

The stomach had contracted considerably, the greater curvature being $1 \frac{1}{2}$ in. above the umbilicus, but the epigastriurn was still somewhat hollow. She had put on flesh, and could eat anything except pork. Since the operation, however, she has had five or six attacks of sickness, the last being five months ago.

Case v.

Mrs. W., aged 43, had suffered with gastric pain, vomiting, and haematemesis for seven years. There was distinct gastro-

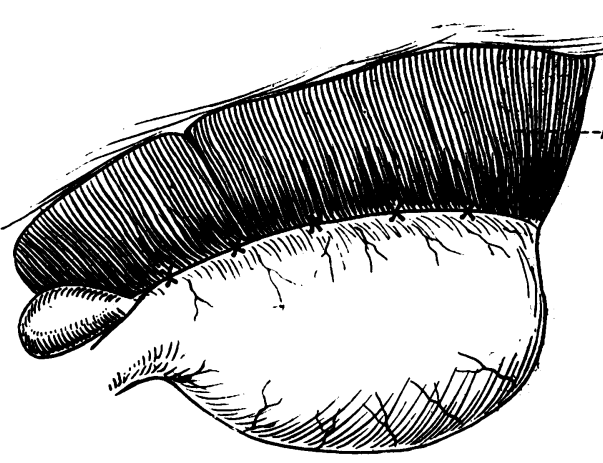

ptosis, the pancereas being felt above the lesser curvature. Gastric uleer with gastrodiagnoged.

Operation. Posterior
gastro-enterostomy was performed, there being distinct evidence of a Fig. 4.-Liver turned up. Sutures shown in Fig. 3 eated ulcer on the legser curvature near its pyloric end. This patient was last seenser months after the operation, when she had put on weight and could eat anything.

\section{The Operation of Selection.}

There were distinct indications for the performance of gastro-enterostomy in the last two cases, and it would probably have been better to have performed the same operation in Case I. This procedure, however, cannot be considered the operation of choice in gastroptosis, the special reasons for excluding the stomach from the alimentary canal which exist in chronic ulcer being absent.

Dr. Beyea" has given a full account of the various operations performed for the condition under consideration. Duret ${ }^{3}$ of Lisle, in 1894, was the first to treat gastroptosis by an operation, which consisted of suturing the lesser curvature of the stomach to the anterior abdominal parietes just below the ensiform cartilage. This procedure would be impracticable in many cases owing to the position of the left lobe of the liver. Davis adopted a similar procedure, but attached the lesser omentum to the parietes. Rovsing in 3 cases sutured the anterior wall of the stomach by means of three vertically-placed rows of interrupted sutures to the anterior abdominal parietes. Coffey ${ }^{6}$ sutured the great omentum below its attachment to the transverse colon to the anterior abdominal wall. Beyea $^{7}$ himself adopted a much more scientific procedure in 4 cases.

Three rows of interrupted silk sutures were introduced so as to plicate and thus shorten the gastro-hepatic and gastrophrenic ligaments in the following manner. The first row, beginning in the gastro-phrenic Jigament, and extending across the gastro-hepatic ligament to almost opposite the across the gastro-hepatic ligament to almost opposite the pyloric orifice and hepatico-duodenal ligament, was introduced so as to form a plication in the centre of these ligaments, and included from above downwards or vertically about $4 \mathrm{~cm}$. of tissue. Five sutures, about 1 in. apart, were introduced from right to left, and caught in haemostatic forceps. The next row of sutures was introduced in the same manner, but $2.5 \mathrm{~cm}$. above and below the first row. Then third row was introduced just above the frastric vessen a short distance below the dia hove and aros was strictly confined to the normal ligamentary supports, and with the length of the ligaments, being greater towards the with the

Bier ${ }^{8}$ of (iriefswald reported 4 cases operated on in a manner very similar to the procedure of Beyea. Three or four sutures were placed through the gastro-hepatic omentum from above downwards so as to form two plications one above the other in the ligament. The first suture attached the serous and muscular coats of the pyloric end of the stomach at the lesser curvature to the capsule of the liver; the others included only the ligament. Also, in two cases, the shortening of the gastrohepatic ligament was supplemented by passing two sutures through the serosa of the lesser curvature of the stomach, and then through the capsule of the left lobe of the liver. From this latter circumstance it would seem that Bier had met with the same difficulty as regards the tenuity of the lesser omentum as myself.

It is evident that our aim should be to raise the stomach into its normal position, if possible by means of its natural suspensory ligaments, the gastrophrenic ligament and gastro-hepatic omentum. This is carried out by Beyea's operation, which would naturally suggest itself to any one looking carefully at the condition of the parts, as it did to myself. But unfortunately, in three of my cases, this procedure was absolutely impracticable on account of the extreme tenuity of the gastro-hepatic omentum; it would scarcely bear touching, much less suturing. Under these circumstances the next best course is to utilize the structure to which the gastro-hepatic omentum is attached, namely, the liver and the stronger portion of the omentum forming its upper root of attachment. Again, the lower sutures must be placed through the serous and muscular eorts of the stomach and the anterior attachment of the omentum, in order to avoid the gastric and pyloric arteries. Such a procedure gives a firm hold to sutures, and at the same time is not likely to interfere with the mobility of the stomach. An important reason for raising the lesser curvature is that owing to the falling of this part of the stomach. and the greater fixation at a higher level of the pylorus, a sudden bend, described in some instances as a kink, has been produced near the pyloric orifice. Hence the necessity of effectively raising the prepyloric portion of the lesser curvature to the level of the orifice.

In my second case there was marked falling of the liver, and this existed to some extent in Case III. The stomach being largely suspended from the liver, this hepatoptosis must clearly be remedied, and the simplest manner of effecting it is by suturing the liver to the costal margin.

In regard to operations of the type first performed by Duret, the obvious objection is that the adhesions to the anterior abdominal wall, if sufficiently firm to support the stomach, may embarrass the motility of the organ, and may possibly be a source of pain. Nevertheless, in the reported cases of patients operated upon in this manner all improved, and some gained weight.

I must express my indebtedness to $\mathrm{Mr}$. Norman Patterson for making, during the course of the operation the above semidiagrammatic drawings.

REFERENCES.

1 Clinical Journal, 1905. 2 Philudelplija Afedical, Journal, vol. ii. 1903, p. 257. ${ }^{3}$ Revuc de Chir., $1890_{\text {. p. }} 430$. 4.W'estern Medical Review, detphia Medicul Journal, Oetober 11 th, 1902. * Op. cit., p. $259 .{ }^{8}$ Peuts.
Zeitsch. f. Chir., Bd. lvi, 1900, p. 374 . 